\title{
An Integrated Contraflow Strategy for Multimodal Evacuation
}

\author{
Jingyi Hua, ${ }^{1}$ Gang Ren, ${ }^{1}$ Yang Cheng, ${ }^{2}$ and Bin $\operatorname{Ran}^{1,2}$ \\ ${ }^{1}$ School of Transportation, Southeast University, No. 2 Sipailou, Nanjing 210096, China \\ ${ }^{2}$ Department of Civil and Environmental Engineering, University of Wisconsin-Madison, 1415 Engineering Drive, \\ Madison, WI 53706, USA \\ Correspondence should be addressed to Gang Ren; rengang@seu.edu.cn
}

Received 15 October 2013; Revised 11 April 2014; Accepted 17 April 2014; Published 13 May 2014

Academic Editor: Hector Puebla

Copyright (C) 2014 Jingyi Hua et al. This is an open access article distributed under the Creative Commons Attribution License, which permits unrestricted use, distribution, and reproduction in any medium, provided the original work is properly cited.

\begin{abstract}
To improve the efficiency of multimodal evacuation, a network aggregation method and an integrated contraflow strategy are proposed in this paper. The network aggregation method indicates the uncertain evacuation demand on the arterial subnetwork and balances accuracy and efficiency by refining the local road subnetworks. The integrated contraflow strategy contains three arterial configurations: noncontraflow to shorten the strategy setup time, full-lane contraflow to maximize the evacuation network capacity, and bus contraflow to realize the transit cycle operation. The application of this strategy takes two steps to provide transit priority during evacuation: solve the transit-based evacuation problem with a minimum-cost flow model, firstly, and then address the autobased evacuation problem with a bilevel network flow model. The numerical results from optimizing an evacuation network for a super typhoon justify the validness and usefulness of the network aggregation method and the integrated contraflow strategy.
\end{abstract}

\section{Introduction}

Emergency evacuation has long been a topic in hazard preparedness and response management. The evacuation planning of advance-notice emergencies (such as hurricane and flood) with long warning time and large geographic scale is a challenging problem because of the unexpected traffic demand, complex road networks, and uncertain driver behaviors. A significant amount of research has been conducted on this problem during the last decade, but most of these studies address automobile evacuation only. The evacuation of the low-mobility population that has little access to personal vehicles, however, has not been fully investigated [1].

Many people fall into the low-mobility population, especially in large cities and developing countries. Evacuation planning should consider their needs; for example, as many as $25-30 \%$ of the population does not have access to personal cars in New Orleans [2]. After the failure of low-mobility population evacuation during Hurricane Katrina, several efforts have been made to improve multimodal evacuation $[3,4]$. Using transportation simulation system TRANSIMS [5], VISSIM [6], and DynusT [7], researchers developed various traffic management frameworks to simulate multimodal evacuation and to evaluate the efficiency of different modes. On the aspect of numerical analysis, Abdelgawad et al. proposed a multimodal optimization framework with three objectives: minimizing in-vehicle travel time, minimizing atorigin waiting time, and minimizing fleet cost [8]. Di Gangi developed a mesoscopic DTA model to introduce simulation of multimodal systems and to enhance some capabilities such as queue spillover and overtaking management [9]. Wang et al. presented a multiple-objective optimization model with the consideration of evacuation priorities and traffic setup time in the multimodal evacuation problem [10].

However, few existing methods have considered the priority of transit. The transit vehicles are supposed to share lanes with other traffic along regular transit routes. Once the low-mobility population is large or the road network is oversaturated, this limitation would impede the cycle operation of transit vehicles. In other words, the overlook of transit priority would lower the evacuation efficiency of lowmobility population.

To address this challenge in evacuation planning, we pursue three objectives in this study: (a) developing a network aggregation method to simplify the loading of the 


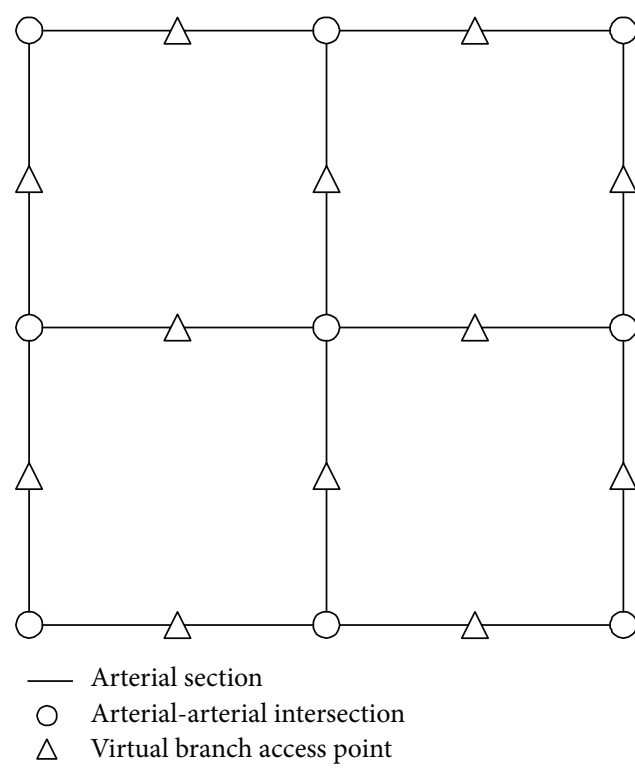

(a) Conventional network representation

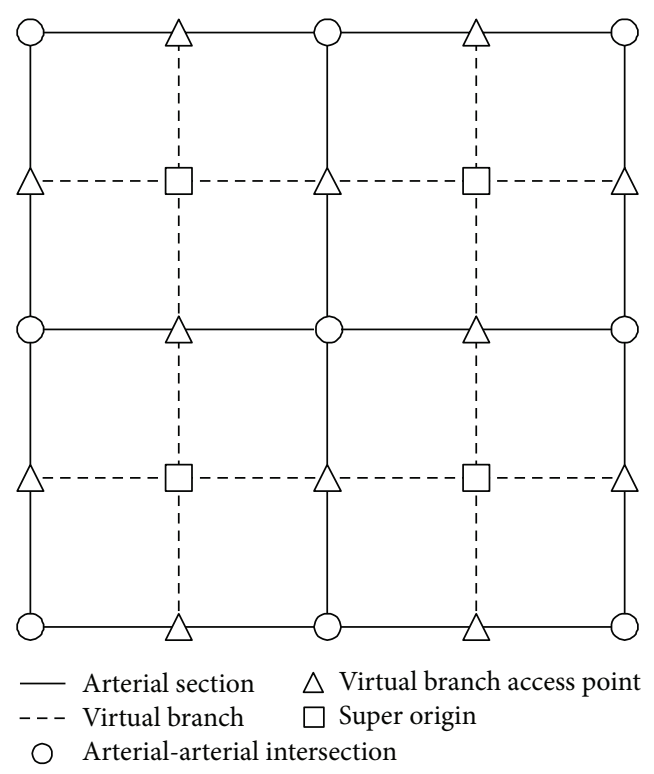

(b) Aggregated network representation

FIGURE 1: Comparison of network representation methods during evacuation.

multimodal evacuation demand; (b) presenting an integrated contraflow strategy to provide priority to transit vehicles; and (c) devising a two-stage evacuation model to the proposed strategy.

The rest of the paper is organized as follows. The next section first presents the network aggregation method and the integrated contraflow method. In the model formulation section, we describe the evacuation models for transit-based evacuees and auto-based evacuees separately. Specific algorithmic designs of our solution method are then elaborated. In the case study section, we test the performance of the proposed method in solving a real evacuation planning problem. And the last section summarizes and concludes with a discussion of future work.

\section{Methodology}

In this paper, the evacuees on the source place are classified into two categories: the auto-based evacuees (i.e., high-mobility population) and the transit-based evacuees (i.e., low-mobility population). The auto-based evacuees are supposed to withdraw the hazardous area using their own vehicles, while the transit-based evacuees may need to be sent to the processing centers within the hazardous area (such as railway stations, airports, and transit hubs) for further evacuation. Based on the analysis of evacuation demands, this section presents a network aggregation method and an integrated contraflow strategy to show how to provide transit priority during multimodal evacuation.

2.1. Network Aggregation Method. Because of the large number of intersections and streets in urban areas, it is impossible to cover the whole urban network in network design problems. It is also a challenge for evacuation network design. Researchers usually abstract arterial subnetwork for optimization design from the whole network. Under this kind of network representation, as shown in Figure 1(a), origin nodes of the network are located on arterials and each node has a fixed evacuation demand. This representation yields two disadvantages during multimodal evacuation. The first disadvantage is that the conventional representation hinders the application of transit priority. The transit-based evacuees are located in each arterial origin node, and it is neither feasible nor cost effective to provide transit priority on every arterial link. The second disadvantage is the inaccurate estimates of the evacuation demand distribution. Evacuees actually live in the zone surrounded by arterials and have the choice of accessing different adjacent arterials; thus, it is very hard to determine the fixed demand at arterial origin nodes. In other words, the evacuation demand of an arterial origin node is usually uncertain. To eliminate these two disadvantages in network representation, a network aggregation method is presented, as shown in Figure 1(b), which aims to reduce the number of origin nodes and balance calculation accuracy and efficiency.

Before proceeding to the details of this aggregation method, it is worthwhile defining the road functional classification during evacuation first. Two basic classes of roads are considered in this study: arterials and local roads. Arterials are defined as road segments without direct connection with origin nodes, and they provide the mobility that evacuees need to reach network destinations as quickly and safely as possible. Local roads are directly connected with origin nodes, and they only serve local traffic access to arterials. Based on this classification, the evacuation network is divided into two parts: an arterial subnetwork and a number of simple local road subnetworks. A local road subnetwork is a closed zone bounded by arterial segments (e.g., the four squares surrounded by solid lines in Figure 1(b)). 
There is another advantage of this network aggregation method: the flexible definition of road classification. If the network is very large or just a rough result is needed, the range of arterials can be restricted to a higher level (e.g., arterials with median barrier). If it is a district network or detailed result is needed, the collectors can also be included in the range of arterials.

Each vehicle follows a similar two-stage evacuation process. First stage is from an origin location (e.g., home) to an arterial access point, within a local road subnetwork. Second stage is from an arterial access point to a destination via the arterial subnetwork. It is obvious that the evacuation distance of the second stage is much longer than that of the first stage, and those measures on arterial subnetwork are cost effective. Thus, we leave this subnetwork unaggregated to ensure the accuracy of the results. In addition, as the travel time on local road has little effect on the evacuation time, a local road subnetwork can then be aggregated to one super origin node and several virtual branches.

The aggregation process works as follows: firstly, a super origin node is set to accommodate the entire evacuation demand in each zone; secondly, a virtual branch access point is used to represent the whole local road access points on an arterial segment (i.e., the road section between two adjacent arterial-arterial intersections); thirdly, virtual branches connecting super origin node with adjacent virtual branch access points are introduced to describe the paths accessed into the relevant arterial segments.

2.2. Integrated Contraflow Strategy. Contraflow is generally the use of one or more lanes of inbound travel for traffic movement in the outbound direction, which increases the operational evacuation capacity [11]. The research regarding contraflow has concentrated on the effectiveness, feasibility, and safety issues of implementing this measure. The research regarding contraflow has concentrated on the effectiveness and feasibility by simulation or numerical analysis. Using microscopic simulation, Theodoulou and Wolshon, Lim and Wolshon, and Williams et al. assessed the adequacy of the contraflow plan in terms of effectiveness or termination design [12-14]. On the aspect of numerical analysis, heuristic algorithms are usually used to solve the evacuation contraflow problems, such as tabu-based [15], greedy-based, and bottleneck relief [16] and genetic algorithm [17]. In actual applications, this measure has been widely used for hurricane evacuation in the states of the U.S. that lie along the Atlantic and Gulf Coasts [18].

However, the conventional contraflow strategy still addressed auto-based evacuation only. Thus, transit vehicles and automobiles were mixed together which reduced the efficiency of transit recycle operation. Concerning this issue, Ren et al. proposed a new variant of contraflow operation, named bus contraflow, for the evacuation of transit-based evacuees [19]. This approach transformed contraflow lanes into exclusive bus lanes during evacuation, which guaranteed transit priority without degrading the efficiency of normal outbound lanes.

Based on the research results above, an integrated contraflow strategy is suggested for the global optimization of multimodal evacuation. Arterials in the evacuation network contain three configurations, as shown in Figure 2, under the control of integrated contraflow strategy: (a) noncontraflow to shorten the strategy setup time; (b) full-lane contraflow to maximize the evacuation network capacity; and (c) bus contraflow to realize the transit cycle operation. The bus contraflow configuration is applied on the arterials serving as evacuation transit routes. For the arterials only serving automobiles, the integrated strategy deploys full-lane contraflow operation to the arterials with oversaturated flow (i.e., the evacuation flow exceeded the normal outbound capacity) and leaves the rest unchanged.

Since there are two kinds of evacuation demands existing in the evacuation network, the application of the integrated contraflow strategy comprises two steps: solve the transitbased evacuation problem, firstly, and then address the auto-based evacuation problem. The detailed optimization procedures are as follows.

Step 1. Transit-based evacuation traffic assignment:

(a) generate the transit-based demands at the super origin nodes;

(b) assign the transit-based evacuation flow according to the selected route choice model;

(c) apply the bus contraflow configuration on the arterial links serving as evacuation transit routes.

Step 2. Auto-based evacuation traffic assignment:

(a) remove the bus contraflow lanes from the network and calculate the remaining capacity;

(b) generate the auto-based demands at the super origin nodes;

(c) assign the auto-based evacuation flow according to the selected route choice model;

(d) determine the configurations of each link (deploy full-lane contraflow operation to the arterial links with oversaturated flow and leave the rest unchanged as noncontraflow).

\section{Model Formulation}

In the integrated contraflow strategy proposed above, appropriate route choice models were needed to assign the transitbased and auto-based evacuation flow. Thus, a group of feasible optimization models are established in this section.

\subsection{Network Notation. See the network notation in Notations} section.

A graph $G=(N, A)$ is defined to represent an aggregated evacuation network, where $N$ and $A$ are the sets of nodes and links, respectively. Both node set $N$ and link set $A$ are made up of their own component parts. The node set $N$ includes four exclusive parts: super origin node set $N_{S}$, virtual access node set $N_{A}$, intersection node set $N_{I}$, and destination node set $N_{D}$, where $N=N_{S} \cup N_{A} \cup N_{I} \cup N_{D}$. Accordingly, the link 


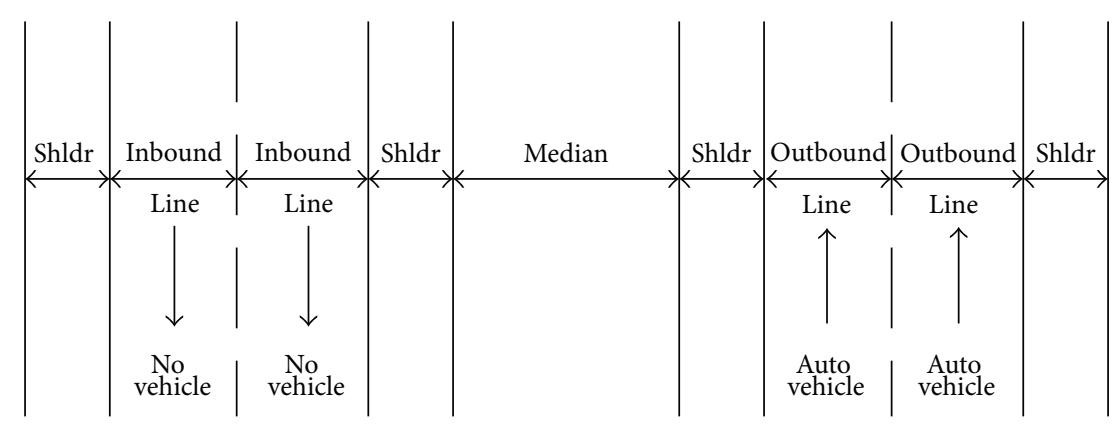

(a) Noncontraflow operation

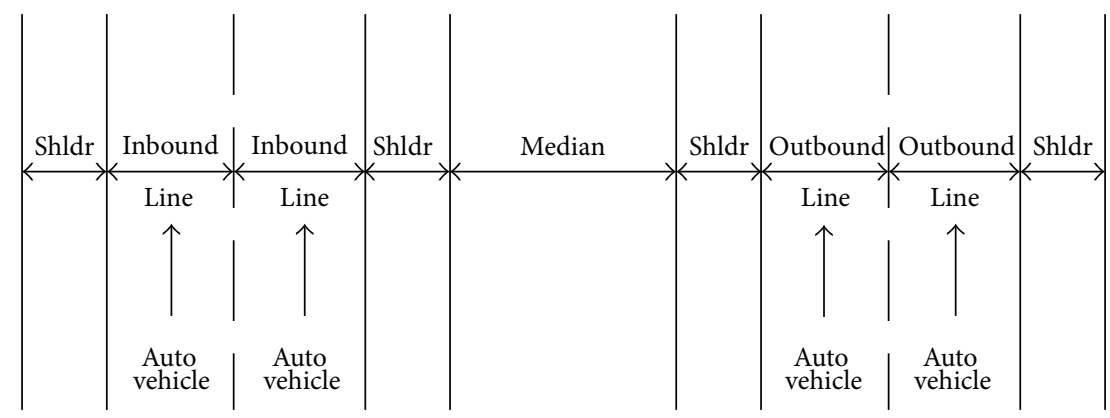

(b) Full-lane contraflow operation

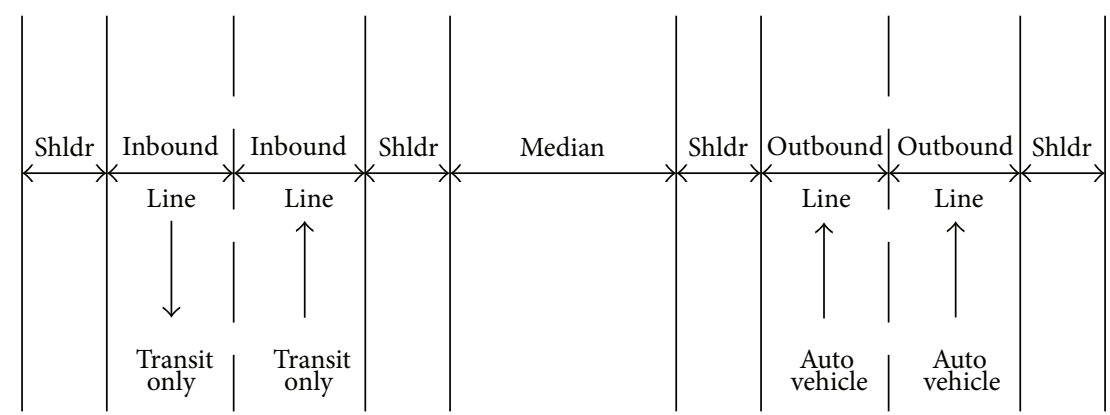

(c) Bus contraflow operation

FIGURE 2: Arterials configurations under the control of integrated contraflow strategy.

set $A$ includes two exclusive parts: roadway-section link set $A_{R}$ and virtual branch link set $A_{B}$, where $A=A_{R} \cup A_{B}$.

As each component, part of node set and link set, has its own characteristics, some assumptions are needed to make definite notation and simplify the calculation. These assumptions can be classified as one of the following four groups:

(i) one-way assumption,

(ii) virtual access assumption,

(iii) link property assumption,

(iv) super destination assumption.

3.1.1. One-Way Assumption. The optimization models proposed by many researchers changed most of the arterials into one-way roads during evacuation $[15,16,20]$. In addition, the research results of Shekhar and Xie proved that 100 percent degree of contraflow (i.e., two-way roads are all converted to one-way roads) was one of the optimum or near optimum solutions to their network design problems $[21,22]$. Therefore, all arterial segments are one-way sections in the evacuation network in this study. This means that the noncontraflow links also operate in one direction and leave the lanes of the opposite direction closed. This assumption can reduce the computation complexity of the proposed models and diminish the confusion of evacuees.

3.1.2. Virtual Access Assumption. An arterial segment is divided into two roadway-section links by the virtual access node, and these two links are assumed to have the same length (i.e., the node is located in the middle of this section). Also, these two links can have opposite operation directions during evacuation.

3.1.3. Link Property Assumption. The link properties are determined by the link functions. A roadway-section link is used for calculating evacuation time; thus, it is treated as an ordinary graphical link, associated with capacity, length, and other travel attributes. A virtual branch link provides access to 
the arterial subnetwork; so its link properties contain capacity and direction but no travel cost.

3.1.4. Super Destination Assumption. Auto-based evacuation usually contains several destination nodes, but this feature increases the difficulty of problem solving. Thus, a single "super" destination node connecting all network exit nodes is established in each evacuation model (i.e., one for transitbased evacuation and another for auto-based evacuation). In this way, all travelers have the same destination but may reach it through different network exits, each of which constitutes a "real" destination.

3.2. Transit-Based Evacuation Model. As the transit-based evacuation is usually managed by the local government, transit drivers have little opportunity to select their evacuation routes. Thus, the routing problem of transit-based evacuation has been considered as a minimum-cost flow problem in this paper. The objective is to route transit vehicles to their closest evacuation destination. The evacuation model has been formulated as follows:

$$
\min Z_{D}=\sum_{(i, j) \in A_{R}} x_{i j}^{*} l_{i j},
$$

subject to

$$
\begin{gathered}
\sum_{j \in \Gamma_{i}} x_{i j}^{*}-\sum_{k \in \Gamma_{i}^{-1}} x_{k i}^{*}=0, \quad \forall i \in N_{A} \cup N_{I} \\
\sum_{i \in \Gamma_{h}} x_{h i}^{*}=d_{h}^{*}, \quad \forall h \in N_{S} \\
x_{i j}^{*} \leq c_{i j}^{*} z_{i j}, \quad \forall(i, j) \in A_{R} \\
0 \leq x_{i j}^{*}, \quad \forall(i, j) \in A .
\end{gathered}
$$

Constraints (2) and (3) describe the flow conservation constraints, meaning that the inflow volume equals the outflow volume. Constraint (4) is used to ensure the proper allowed amount of transit-based flow on each link. When $z_{i j}=1$, it indicates that bus contraflow configuration is applied on link $(i, j)$; otherwise, the link is not serving as evacuation transit routes. Constraint (5) places the lower bound on all transit-based flow variables.

3.3. Auto-Based Evacuation Model. Different from the transit drivers which have fixed route during evacuation, private drivers may be uncertain about the evacuation planning and also may be using unfamiliar routes. Thus, the routing problem of auto-based evacuation is formulated as a discrete network design model where the underlying traffic flow pattern follows a user equilibrium model. It has a bilevel structure. The top level minimizes total network evacuation time using the integrated contraflow strategy, while the traffic volumes and travel times in streets are derived from equilibrium traffic assignment in the bottom level. The model can be described as follows.
Up-level model

$$
\min Z_{T}=\sum_{(i, j) \in A_{R}} x_{i j} t_{i j},
$$

where

$$
\begin{gathered}
t_{i j}=t_{i j}^{0}\left(1+\alpha\left(\frac{x_{i j}}{c_{i j}^{\prime}}\right)^{\beta}\right), \quad \forall(i, j) \in A_{R}, \\
c_{i j}^{\prime}=c_{i j}-c_{i j}^{*} z_{i j}
\end{gathered}
$$

subject to

$$
\begin{gathered}
y_{i j}+y_{j i} \leq 1, \quad \forall(i, j),(j, i) \in A_{R} \\
x_{i j} \leq c_{i j}^{\prime} y_{i j}, \quad \forall(i, j) \in A_{R} \\
0 \leq x_{i j}, \quad \forall(i, j) \in A .
\end{gathered}
$$

Low-level model

$$
\min Z_{U}=\sum_{(i, j) \in A_{R}} \int_{0}^{x_{i j}} t_{i j}(\omega) d \omega,
$$

subject to

$$
\begin{gathered}
d_{p q}=\sum_{r \in R_{p q}} X_{r}^{p q}, \quad \forall(p, q) \in V, \\
x_{i j}=\sum_{(p, q) \in V} \sum_{r \in R_{p q}} X_{r}^{p q} \delta_{i j, r}, \quad \forall(i, j) \in A_{R} \\
X_{r}^{p q} \geq 0, \quad \forall r \in R_{p q} .
\end{gathered}
$$

Equation (7) calculates the travel time and the remaining capacity for auto-based evacuation of each link. Constraint (8) is a contraflow constraint, and it restricts that arterial segments are all converted to one-way sections. The connectivity indicator, $y_{i j}$, for all $(i, j) \in A_{R}$, is a 0-1 dummy variable. Constraint (9) shows the inherent relationship between the connectivity indicator of a link and its auto-based evacuation flow. When $y_{i j}=1$, it indicates that a positive flow on link $(i, j)$ is allowed; when $y_{i j}=0$, it indicates that link $(i, j)$ is blocked and accordingly $x_{i j}=0$. Constraints (10) and (14) place the lower bound on all auto-based evacuation flow variables. Constraints (12) and (13) describe the inherent relationships of evacuation flow rates at the link, path, and $\mathrm{O}-$ D levels.

\section{Solution Method}

According to the methodological framework of integrated contraflow strategy, we develop a two-stage solution method to tackle the proposed optimization models. Firstly, the transit-based evacuation problem is solved using network branch and bound method; then, genetic algorithm (GA) is applied to search for the optimal or approximate optimal network configuration for auto-based evacuation. In this section, the detailed descriptions of algorithms are presented. 
The transit-based evacuation problem can be seen as a minimum-cost flow problem with multiple origin nodes and single super destination node. This is a mixed-integer linear programming problem that can be solved in a very efficient manner using the branch and bound method.

The auto-based evacuation model has a bilevel structure, which is usually solved using heuristic algorithms [23]. GA is a population-based metaheuristic algorithm inspired by the genetic evolution process of species in the nature. It has been widely used to solve the one-way or contraflow design problem, Drezner and Salhi, Zargari and Taromi, and Karoonsoontawong and Lin have applied GA with various fitness functions in their studies [17, 24, 25]. Based on the general procedure of GA, four specific elements of the GAbased approach are introduced.

4.1. Solution Encoding. Discrete decision variables (i.e., link directions) are coded as chromosomes. The chromosomes are 2-row matrices, in which columns correspond to network links and rows correspond to their directions. When the binary variable is 1 , it means that this link direction can be used by evacuation vehicles. Otherwise, it means that this link direction is closed. The length of each chromosome is equal to the total number of roadway-section links and intersection links.

4.2. Fitness Function. Since the purpose of this project is to minimize the total evacuation time for auto-based evacuees, this purpose function could be used as the fitness function. Therefore, the fitness function of this project is considered as a corresponding subproblem for a fixed set of $y_{i j}^{*}$.

Up-level model

$$
\min Z_{T}^{\prime}=\sum_{(i, j) \in A_{R}} x_{i j} t_{i j},
$$

subject to constraint (10)

$$
x_{i j} \leq c_{i j}^{\prime} y_{i j}^{*}, \quad \forall(i, j) \in A_{R} .
$$

Low-level model

$$
\min Z_{U}=\sum_{(i, j) \in A_{R}} \int_{0}^{x_{i j}} t_{i j}(\omega) d \omega
$$

subject to constraints (12)-(14).

$y_{j s}^{*}$ is a fixed set of link connectivity indicator variables represented by a chromosome. The above subproblem can be solved using a sensitivity analysis based (SAB) algorithm which is very common in Network Design Problems with continuous variable. Since the objective function of UM has a linear form, the optimal solution is located at the boundary of feasible space. Therefore, the optimal value of the object is located where all constraints in (16) are satisfied and further increasing its value will make at least one of the constraints infeasible. For each objective value of up-level model, the user equilibrium assignment problem (LM) must be solved to find the equilibrium values. The convex nonlinear problem (LM) can be solved by a convex combinations based algorithm called Frank-Wolfe method, which is often used in NDP studies. For more detailed information, please refer to [26].
4.3. Crossover Operator. Because of the connectivity demand of the network, we adopted the crossover operator developed by Drezner and Wesolowsky [27]. The operator attempts to merge two parents in a way in which the set of links taken from each parent forms a connected set. Such a connected set is built by selecting one node in the network as the "pivot" node. For each pivot node, each link of the network is endowed with a count which represents the minimum number of links needed to get to pivot node. Then, the design of links with a count below the median is taken from one parent, while the design of links with a count above the median is selected from another parent. Based on the partitioning method described, the connectivity of two half networks can be inherited by the next generation. For more detailed information, please refer to [23, 27].

4.4. Connectivity Checking. Two types of network connectivity are checked in our study. Equation (18) checks the node connectivity by judging whether each node has at least one outgoing link. If the first test is successful, the network is subjected to complete connectivity checking using the shortest path generation method between each super origin node and the super destination node. If the shortest path cannot be generated, the network is considered as disconnected. Here, Dijkstra's method is used as the shortest path generation method:

$$
\sum_{j \in \Gamma_{j}} y_{i j}^{*} \geq 1, \quad \forall i \in N_{A} \cup N_{I} .
$$

\section{Case Study}

To have a better understanding of the methods and models, it is necessary to carry out experiments in networks with realistic topology and size. Ningbo City, located on the east coast of the Pacific Ocean, is selected for the case study. This coastal city suffers an average of 3.1 typhoons each year. The evacuation network covers the low-lying areas of Ningbo City, which is approximately $10 \mathrm{~km}$ by $14 \mathrm{~km}$ in size.

The evacuation destinations are set according to the Antityphoon Action Plan of Ningbo City [28]. The destinations of auto-based evacuation are the three access points to the highway system. Since the low-mobility population is planned to be evacuated using high-speed rail, the railway station is identified as the destination of transit-based evacuation.

Using the proposed network aggregation method, the evacuation network is represented in Figure 3. This aggregated network contained 1 transit-based evacuation destination node, 3 auto-based evacuation destination nodes, 18 super origin nodes, 22 arterial-arterial intersections, and 39 roadway sections. The network geometric data (such as section length, number of lanes, and design speed) was set according to the Urban Traffic Planning of Beilun District (2010-2020) [29].

By assuming a typhoon red warning in which all residents in the study area are required to evacuate, thus, a total of 350,000 people are estimated to be evacuated using 69,000 auto vehicles and a number of transit vehicles. Detailed to each zone in Figure 3, the population and the number of 


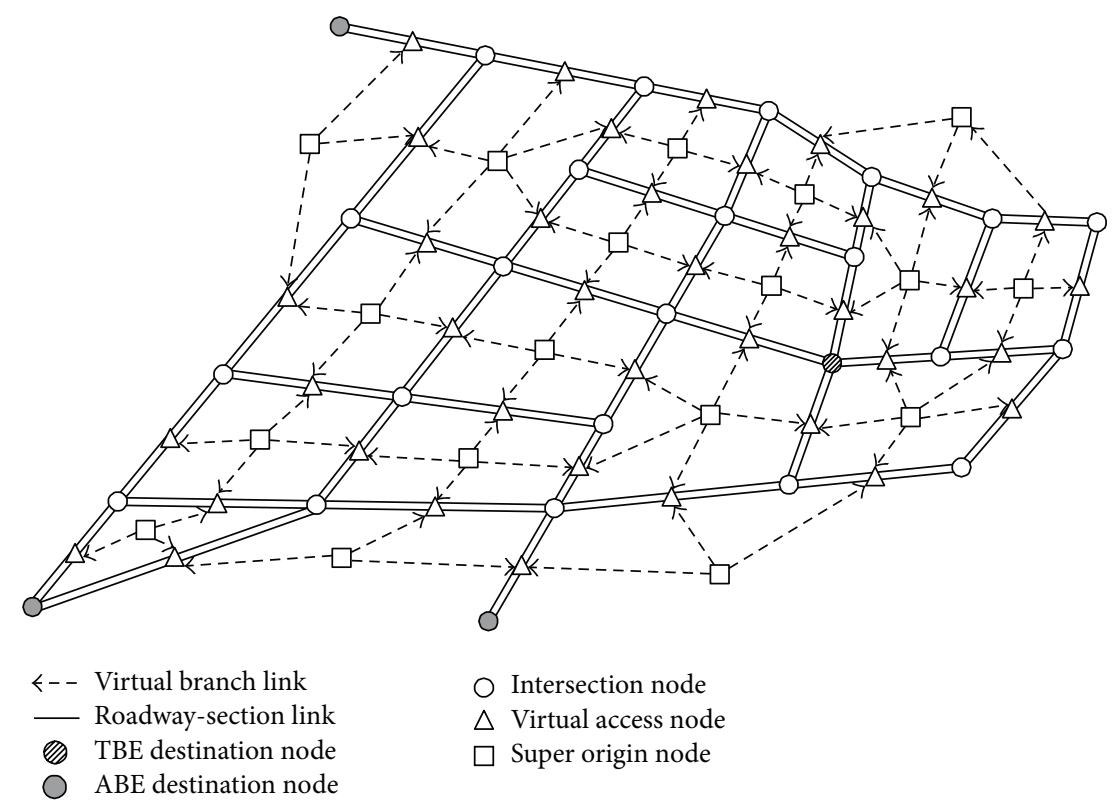

FIGURE 3: Evacuation network of Ningbo City study area.

auto vehicles can be calculated using the data provided by the Urban Traffic Planning of Beilun District (2010-2020) [29].

Both transit-based and auto-based evacuation demands are assumed to be concentrated in super origin nodes. The auto-based demand can be set to the total number of vehicles in the zone directly, while the transit-based demand needs to be calculated as shown in formulation (17). In the case study, the parameter $a$ is set to 2.9 (the average number of family members in the cities of China) and the parameter $b$ is set to 35 (the general number of seats for 12-meter bus). The surge demand rates are approximated by using the historical diurnal curves of evacuation demand generation, such as those presented by Wang et al. [10]. Consider

$$
V_{\text {tran }}^{i}=\frac{P^{i}-a V_{\text {auto }}^{i}}{b},
$$

where $V_{\text {tran }}^{i}$ is the transit-based evacuation demand of zone $i, V_{\text {auto }}^{i}$ is the auto-based evacuation demand of zone $i, P^{i}$ is the number of residents of zone $i$, a is a parameter, the number of occupants of auto vehicles, and $b$ is a parameter, the number of occupants of transit vehicles.

The proposed solution algorithm was implemented by Matlab. Firstly, the transit-based evacuation problem was solved using branch and bound method; then, GA was applied in the auto-based evacuation problem. As GA is one of the heuristic algorithms, the Matlab procedure of the second step was run five times to find a better solution. The best solution of the five runs was then selected as the final plan which was used for the result analysis summarized below.

Figure 4 shows the transit-based evacuation (TBE) plan of the proposed network. The TBE problem has been formulated as a minimum-cost flow model in the paper. For each super origin node, one virtual branch has been selected as the access link of transit vehicles, as this branch can provide the shortest path to the TBE destination among the connected virtual branches. In the arterial subnetwork, those shortest paths merge into several evacuation transit routes which can realize the transit cycle operation. And the bus contraflow configuration is applied to the arterial links serving as evacuation transit routes.

The next step is applying the bilevel auto-based evacuation $(\mathrm{ABE})$ model to the remaining network. To ensure convergence of results, the population number and the iteration number of GA were set to 50 and 300, respectively. And due to the trade-off between solution quality and computational effort, the number of "pivot" nodes in each generation was chosen to be equal to $1 / 5$ of the population with random selection. Details of convergence levels to optimal or approximate optimal solution and the best solution of modeling are highlighted in Figure 5. Inspection of results displayed in Figure 5 reveals that, after about 120 iterations, the model solution reached convergence.

By loading the network reconfiguration results of $\mathrm{ABE}$ into Figure 2, we can get the optimal or approximate optimal evacuation plan for multimodal evacuation, as shown in Figure 6. For each local road subnetwork, the traffic flow on virtual branches is related to the condition of adjacent arterials, and some branches may have a zero-flow rate. This phenomenon shows the uncertain evacuation demand of the arterial subnetwork. On the other hand, the full-lane contraflow operation is deployed on the arterials close to ABE destinations, which can be used to determine the terminals of full-lane contraflow operation in the practice.

\section{Conclusions and Future Work}

During an evacuation, car owners need evacuation traffic management, whereas transit-dependent residents need evacuation transportation support [30]. Thus, an evacuation traffic management method provides more efficient evacuation by considering both car-based and transit-based 


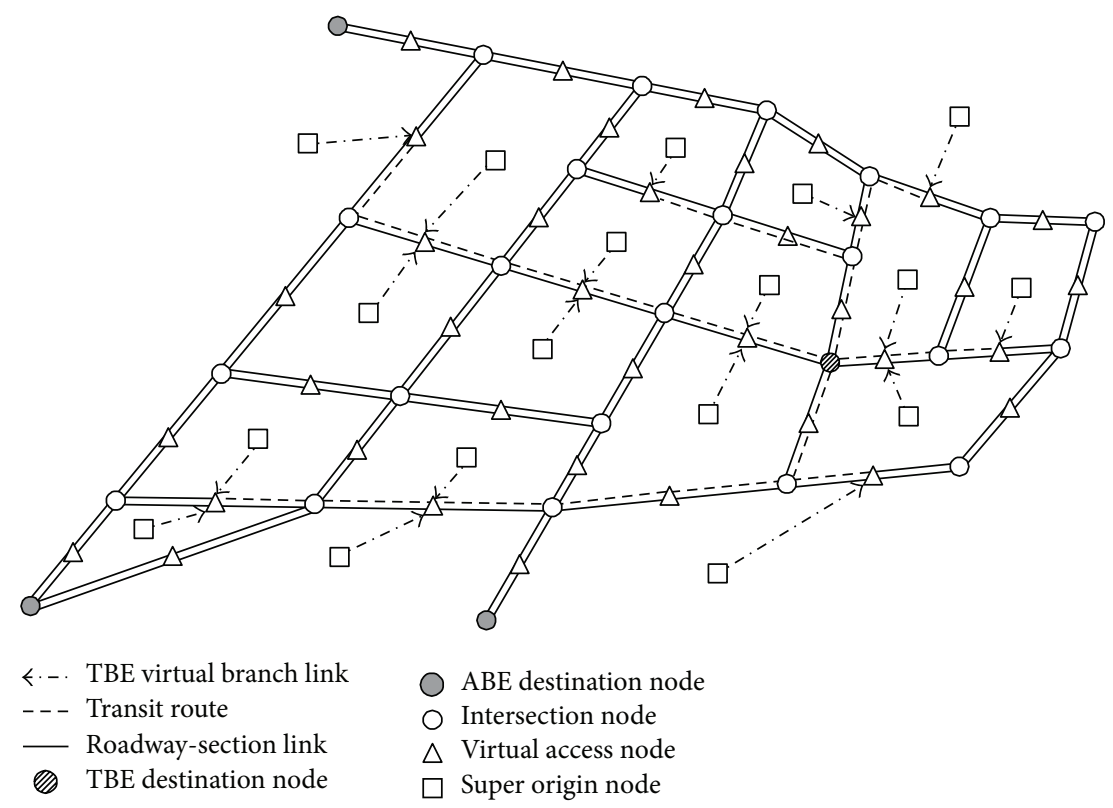

FIGURE 4: Optimal route planning for transit-based evacuation.

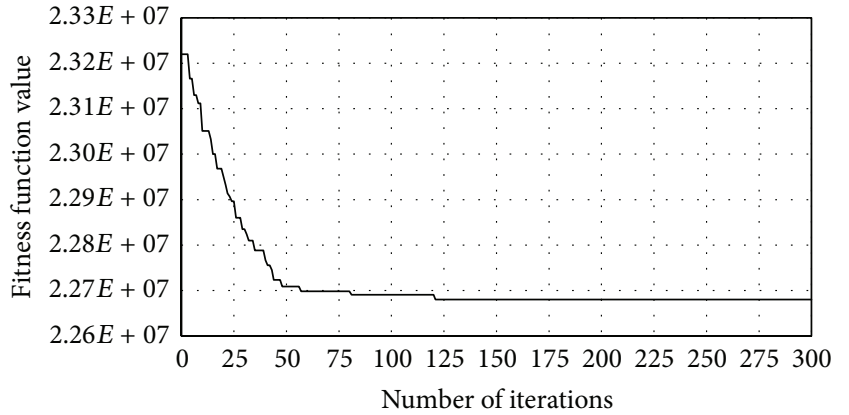

FIGURE 5: Details of convergence levels to optimal or approximate optimal solution.

evacuation needs. With this purpose in mind, this study presents an integrated contraflow strategy to improve the efficiency of multimodal evacuation.

The integrated contraflow strategy is based on the phenomenon that the transit-based evacuees and the autobased evacuees will be evacuated to different destinations. The former are supposed to withdraw the hazardous area using their own vehicles, and the latter may need to be sent to the processing centers within the hazardous area for further evacuation. The integrated contraflow strategy takes a two-step procedure to realize the multimodal optimization: solve the transit-based evacuation problem, firstly, and then address the auto-based evacuation problem.

The two-step optimization procedure was then modeled. As the transit-based evacuation is usually managed by the local government, transit drivers have little opportunity to select their evacuation routes. Thus, the transit-based evacuation model was formulated as a minimum-cost flow model. On the other hand, private drivers may be uncertain about their destination, a bilevel network flow model was then developed for auto-based evacuation. The optimization or approximate optimal evacuation plan of the evacuation network was obtained in the top level, while the traffic volumes and travel times in streets were derived from equilibrium traffic assignment in the bottom level.

And to better reflect the real world evacuation, a new network aggregation method was also described in this study. The arterial subnetwork was a large-scale network with uncertain evacuation demand, as evacuees actually have the choice of accessing adjacent arterials through the local road subnetwork. However, it was impossible to optimize an evacuation network containing all arterials and local roads. The network aggregation method in this study was a balance between accuracy and efficiency. The arterial subnetwork was left unaggregated to ensure the accuracy of the results, while the local road subnetworks were aggregated to simplify the representation and calculation. This method also gathered transit-based evacuation demand of each zone, which reduced the number of origin nodes to accommodate the application of transit priority.

There are a number of directions to pursue in the context of this study. The routing problem in the local road subnetwork should be analyzed in the future. The management of arterial-arterial intersection can also be discussed. For example, the crossing-conflicts are expected to be eliminated to improve the evacuation effectiveness and safety. Finally, the transit priority at intersection may also be considered for the further improvement of the transit-based evacuation.

\section{Notations}

Sets

$N$ : Set of nodes

$N_{S}$ : Set of super origin nodes of the zones

$N_{A}$ : Set of virtual access nodes (virtual branch access point) of the arterials 


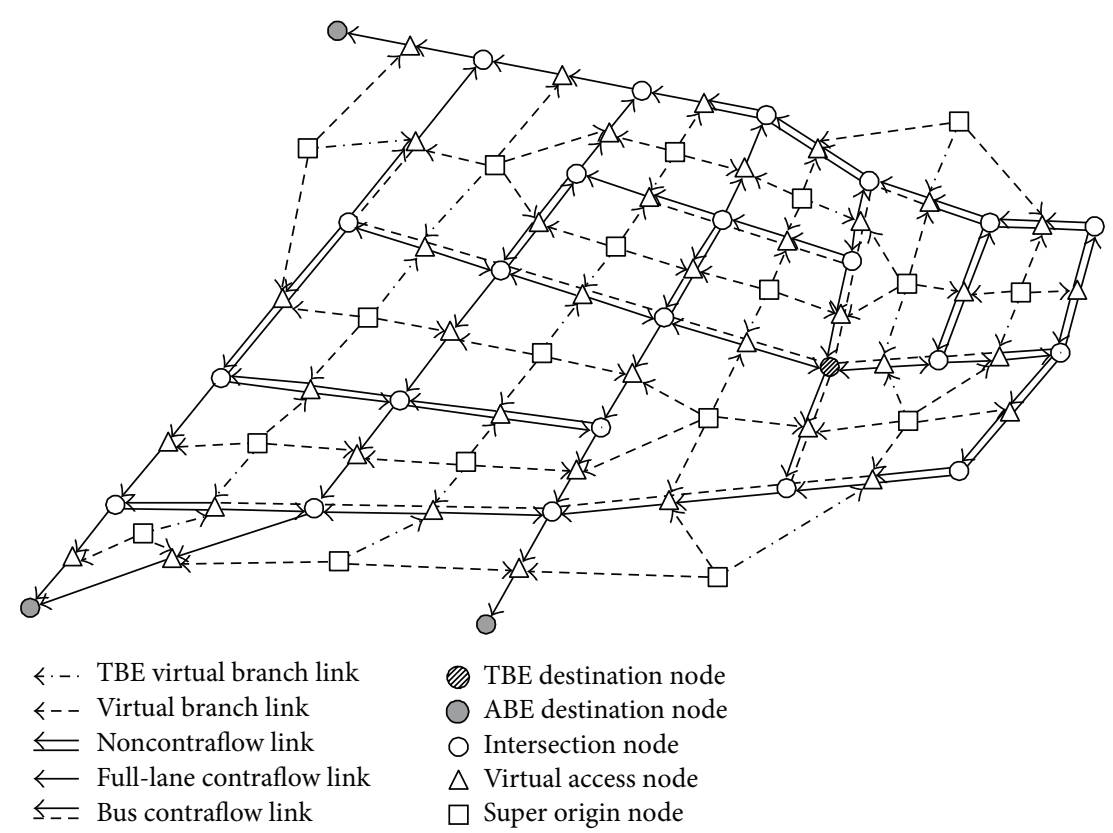

FIGURE 6: Optimal or approximate optimal evacuation plan for multimodal evacuation.

$N_{I}$ : Set of intersection nodes

$N_{D}$ : Set of destination nodes

$\Gamma_{j}$ : Set of downstream nodes of node $j, \forall j \in N$

$\Gamma_{j}^{-1}$ : Set of upstream nodes of node $j, \forall j \in N$

A: Set of links

$A_{R}$ : Set of roadway-section links

$A_{B}$ : Set of virtual branch links

$V: \quad$ Set of O-D pairs

$R_{p q}:$ Set of routes between O-D pair $(p, q), \forall(p, q) \in V$.

\section{Parameters}

$c_{i j}$ : Full-lane reversal capacity of link $(i, j), \forall(i, j) \in A_{R}$

$c_{i j}^{*}$ : Bus contraflow capacity of link $(i, j), \forall(i, j) \in A_{R}$

$l_{i j}$ : Length of link $(i, j), \forall(i, j) \in A_{R}$

$t_{i j}^{0}$ : Free-flow travel time on link $(i, j), \forall(i, j) \in A_{R}$

$d_{h}$ : Auto-based demand generation rate from node $h$, $\forall h \in N_{s}$

$d_{h}^{*}$ : Transit-based demand generation rate from node $h$, $\forall h \in N_{s}$.

\section{Variables}

$Z_{D}$ : Total evacuation distance of transit-based vehicles

$Z_{T}$ : Total evacuation time of auto-based vehicles

$Z_{U}$ : Largest evacuation time of single auto-based vehicle in the road network

$c_{i j}^{\prime}$ : Adjusted capacity of link $(i, j), \forall(i, j) \in A_{R}$

$d_{p q}$ : Evacuation flow rate between O-D pair $(p, q)$, $\forall(p, q) \in V$

$t_{i j}$ : Travel time on link $(i, j), \forall(i, j) \in A_{R}$ $x_{i j}$ : Auto-based evacuation flow rate on link $(i, j)$, $\forall(i, j) \in A$

$x_{i j}^{*}$ : Transit-based evacuation flow rate on link $(i, j)$, $\forall(i, j) \in A$

$X_{r}^{p q}$ : Evacuation flow rate on path $r$ between O-D pair $(p, q), \forall(r) \in R_{p q}, \forall(p, q) \in V$

$y_{i j}$ : Connectivity indicator of link $(i, j), \forall(i, j) \in A_{R}$, where $y_{i j}=0$ or 1

$z_{i j}$ : Bus contraflow indicator of link $(i, j), \forall(i, j) \in A_{R}$, where $z_{i j}=0$ or 1

$\delta_{i j, r}$ : Path-link incidence indicator, where $\delta_{i j, r}=0$ or 1 .

\section{Conflict of Interests}

The authors declare that they have no conflict of interests regarding to the publication of this paper.

\section{Acknowledgments}

This research is supported by the National Natural Science Foundation of China (no. 51078086 and no. 51278101) and the Scientific Research Foundation of Graduate School of Southeast University. The authors appreciate the Jiangsu Provincial Key Laboratory of Transportation Planning and Management of Southeast University.

\section{References}

[1] D. B. Hess and J. C. Gotham, "Multi-modal mass evacuation in upstate New York: a review of disaster plans," Journal of Homeland Security and Emergency Management, vol. 4, no. 3, article 11, pp. 1547-7355, 2007. 
[2] E. Urbina and B. Wolshon, "National review of hurricane evacuation plans and policies: a comparison and contrast of state practices," Transportation Research A: Policy and Practice, vol. 37, no. 3, pp. 257-275, 2003.

[3] J. L. Renne, T. W. Sanchez, and T. Litman, "National study on carless and special needs evacuation planning: a literature review," Planning and Urban Studies Reports and Presentations, 2008.

[4] TRB, "The role of transit in emergency evacuation," Special Report 294, 2008.

[5] H. Naghawi and B. Wolshon, "Transit-based emergency evacuation simulation modeling," Journal of Transportation Safety and Security, vol. 2, no. 2, pp. 184-201, 2010.

[6] L. D. VanLandegen and X. Chen, "Microsimulation of largescale evacuations utilizing metrorail transit," Applied Geography, vol. 32, no. 2, pp. 787-797, 2012.

[7] H. Abdelgawad and B. Abdulhai, "Managing large-scale multimodal emergency evacuations," Journal of Transportation Safety and Security, vol. 2, no. 2, pp. 122-151, 2010.

[8] H. Abdelgawad, B. Abdulhai, and M. Wahba, "Multiobjective optimization for multimodal evacuation," Transportation Research Record, no. 2196, pp. 21-33, 2010.

[9] M. Di Gangi, "Modeling evacuation of a transport system: application of a multimodal mesoscopic dynamic traffic assignment model," IEEE Transactions on Intelligent Transportation Systems, vol. 12, no. 4, pp. 1157-1166, 2011.

[10] J. W. Wang, H. F. Wang, W. J. Zhang, W. H. Ip, and K. Furuta, "Evacuation planning based on the contraflow technique with consideration of evacuation priorities and traffic setup time," IEEE Transactions on Intelligent Transportation Systems, vol. 14, no. 1, pp. 480-485, 2013.

[11] B. Wolshon, "One-way-out: contraflow freeway operation for hurricane evacuation," Natural Hazards Review, vol. 2, no. 3, pp. $105-112,2001$.

[12] G. Theodoulou and B. Wolshon, "Alternative methods to increase the effectiveness of freeway contraflow evacuation," Transportation Research Record, no. 1865, pp. 48-56, 2004.

[13] E. Lim and B. Wolshon, "Modeling and performance assessment of contraflow evacuation termination points," Transportation Research Record, no. 1922, pp. 118-128, 2005.

[14] B. M. Williams, A. P. Tagliaferri, S. S. Meinhold, J. E. Hummer, and N. M. Rouphail, "Simulation and analysis of freeway lane reversal for coastal hurricane evacuation," Journal of Urban Planning and Development, vol. 133, no. 1, pp. 61-72, 2007.

[15] H. Tuydes and A. Ziliaskopoulos, "Tabu-based heuristic approach for optimization of network evacuation contraflow," Transportation Research Record, no. 1964, pp. 157-168, 2006.

[16] S. Kim, S. Shekhar, and M. Min, "Contraflow transportation network reconfiguration for evacuation route planning," IEEE Transactions on Knowledge and Data Engineering, vol. 20, no. 8, pp. 1115-1129, 2008.

[17] A. Karoonsoontawong and D. Y. Lin, "Time-varying lane-based capacity reversibility for traffic management," Computer-Aided Civil and Infrastructure Engineering, vol. 26, no. 8, pp. 632-646, 2011.

[18] C. Xie, D. Y. Lin, and S. Travis Waller, "A dynamic evacuation network optimization problem with lane reversal and crossing elimination strategies," Transportation Research E: Logistics and Transportation Review, vol. 46, no. 3, pp. 295-316, 2010.

[19] G. Ren, J. Hua, Y. Chen, Y. Zhan, and B. Ran, "Bus contraflow lane: improved contraflow approach in freeway evacuation,"
Transportation Research Record: Journal of the Transportation Research Board, vol. 2312, pp. 150-158, 2012.

[20] G. Kalafats and S. Peeta, "Planning for evacuation: insights from an efficient network design model," Journal of Infrastructure Systems, vol. 15, no. 1, pp. 21-30, 2009.

[21] C. Xie and M. A. Turnquist, "Lane-based evacuation network optimization: an integrated Lagrangian relaxation and tabu search approach," Transportation Research C: Emerging Technologies, vol. 19, no. 1, pp. 40-63, 2011.

[22] S. Shekhar and S. Kim, "Contraflow transportation network reconfiguration for evacuation route planning," Tech. Rep. MN/RC-2006-21, Department of Computer Science and Engineering, University of Minnesota, 2006.

[23] E. Miandoabchi and R. Z. Farahani, "Optimizing reserve capacity of urban road networks in a discrete Network Design Problem," Advances in Engineering Software, vol. 42, no. 12, pp. 1041-1050, 2011.

[24] Z. Drezner and S. Salhi, "Using hybrid metaheuristics for the one-way and two-way network design problem," Naval Research Logistics, vol. 49, no. 5, pp. 449-463, 2002.

[25] S. A. Zargari and R. Taromi, "Selecting an optimum configuration of urban one-way and two-way streets using genetic algorithms," International Journal of Civil Engineering, vol. 4, no. 3, pp. 244-259, 2006.

[26] Z. Gao, J. Wu, and H. Sun, "Solution algorithm for the bi-level discrete network design problem," Transportation Research B: Methodological, vol. 39, no. 6, pp. 479-495, 2005.

[27] Z. Drezner and G. O. Wesolowsky, "Network design: selection and design of links and facility location," Transportation Research A: Policy and Practice, vol. 37, no. 3, pp. 241-256, 2003.

[28] Ningbo People's Government Office, Anti-Typhoon Action Plan of Ningbo City, Local Government Planning, Ningbo, China, 2008.

[29] Ningbo Planning Bureau, Urban Traffic Planning of Beilun District (2010-2020), Local Government Planning, Ningbo, China, 2007.

[30] X. Zhang, Q. Shi, R. He, and B. Ran, "Network emergency evacuation modeling: a literature review," in Proceedings of the 2010 International Conference on Optoelectronics and Image Processing, pp. 30-34, Haiko, China, November 2010. 


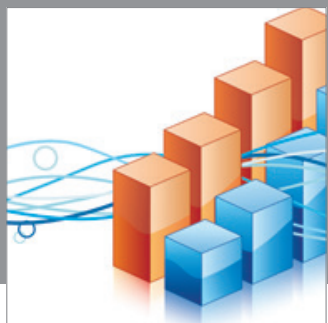

Advances in

Operations Research

mansans

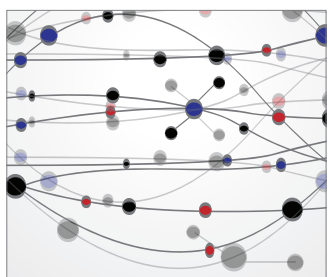

The Scientific World Journal
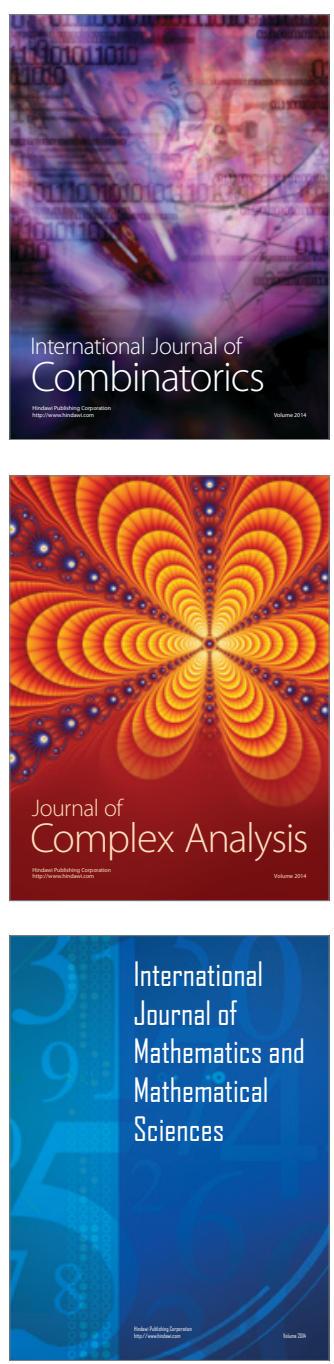
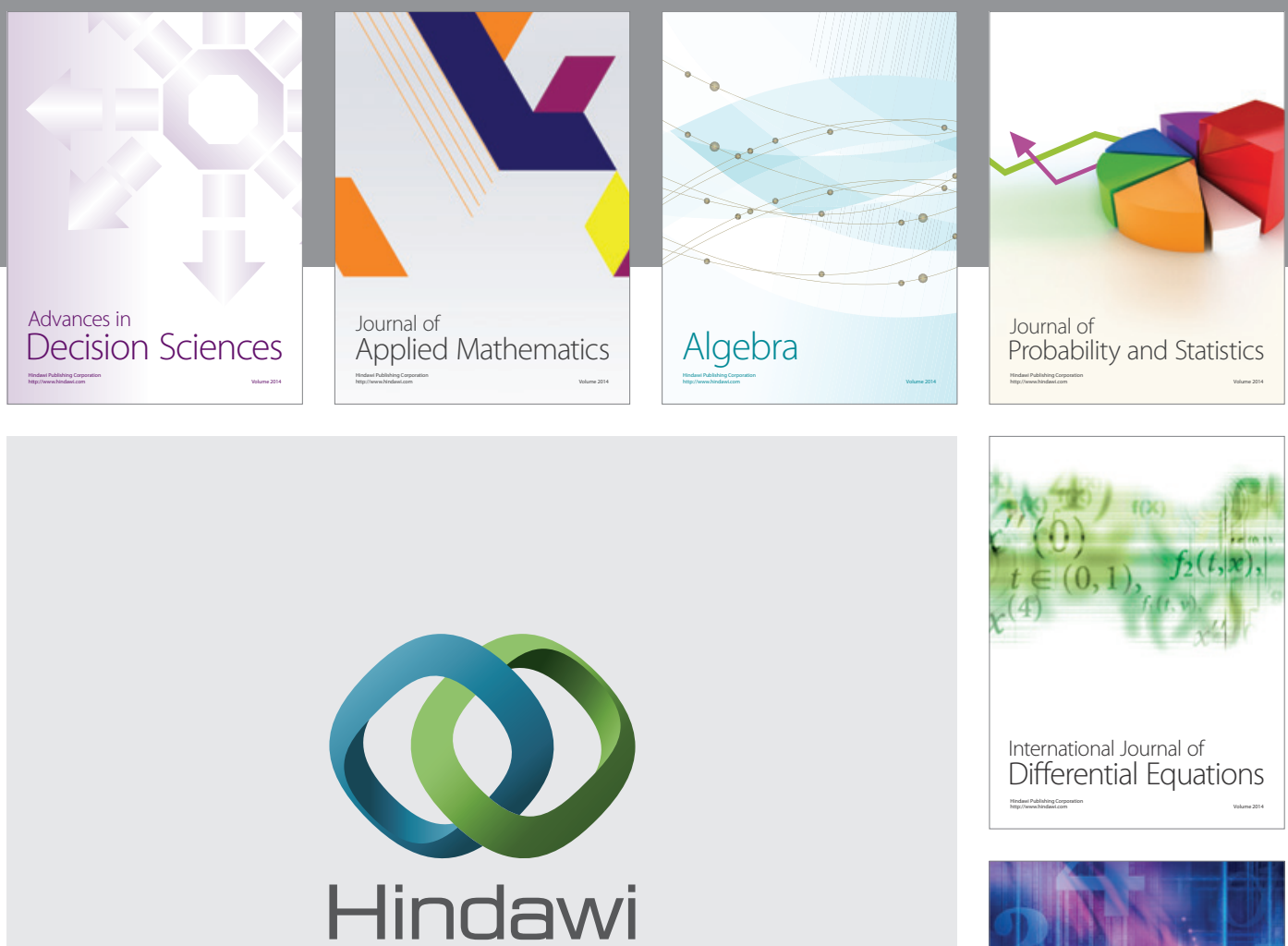

Submit your manuscripts at http://www.hindawi.com
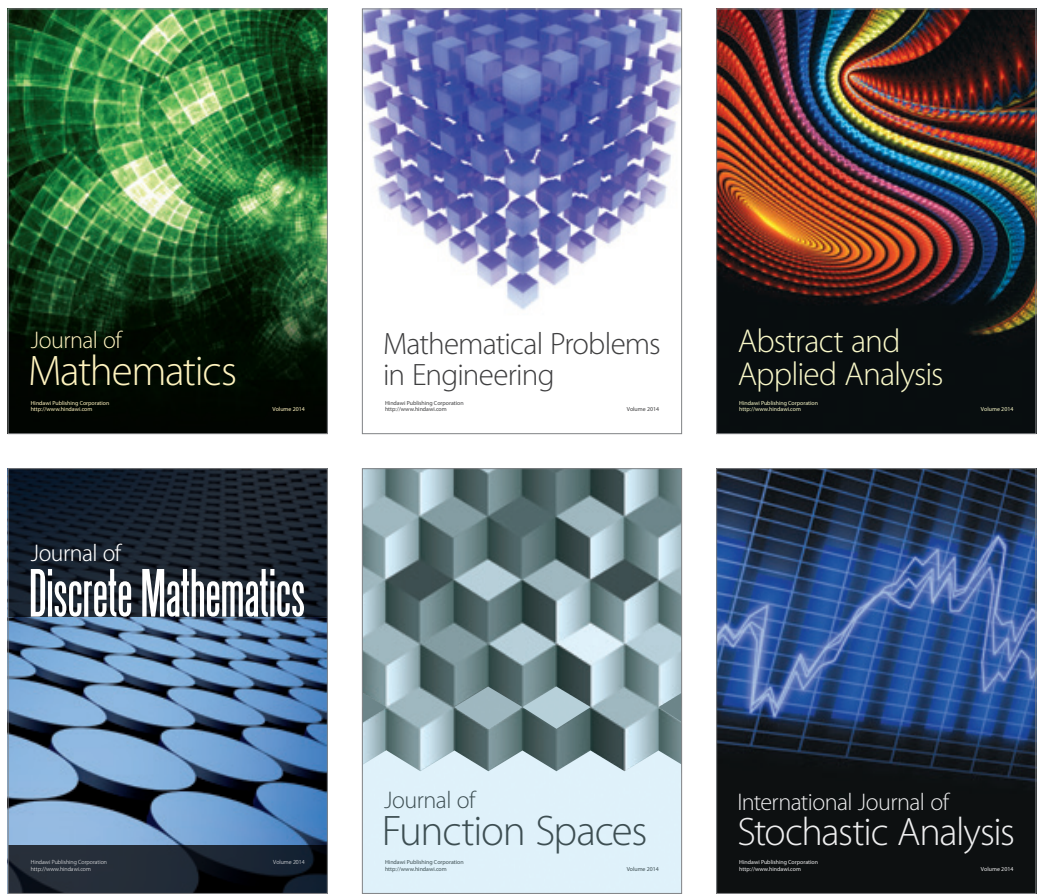

Journal of

Function Spaces

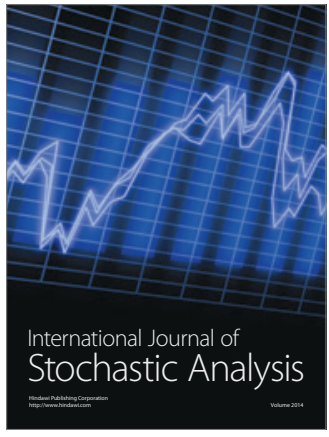

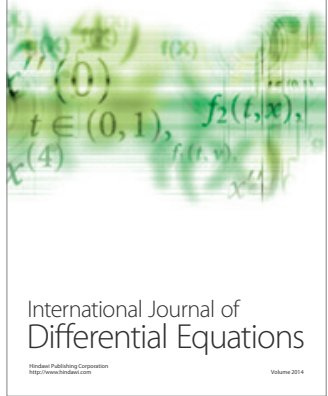
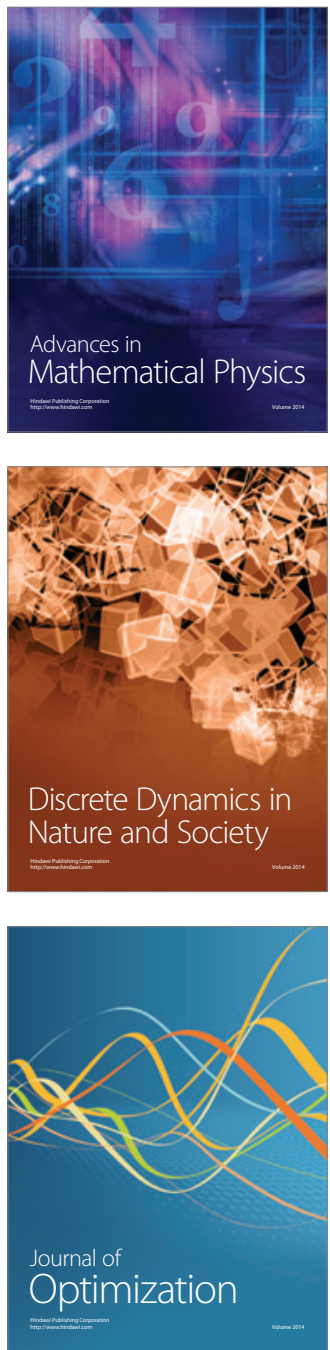